$12-1-1988$

\title{
Cooperative Effects Among Partial Waves in Mie Scattering
}

James A. Lock

Cleveland State University, j.lock@csuohio.edu

Follow this and additional works at: https://engagedscholarship.csuohio.edu/sciphysics_facpub

Part of the Physics Commons

How does access to this work benefit you? Let us know!

\section{Publisher's Statement}

This paper was published in Journal of the Optical Society of America A: Optics Image Science and Vision and is made available as an electronic reprint with the permission of OSA. The paper can be found at the following URL on the OSA website: http://www.opticsinfobase.org/josaa/ abstract.cfm?URI=josaa-5-12-2032. Systematic or multiple reproduction or distribution to multiple locations via electronic or other means is prohibited and is subject to penalties under law.

\section{Original Citation}

Lock, James A. "Cooperative Effects Among Partial Waves in Mie Scattering." Journal of the Optical Society of America A: Optics Image Science and Vision 5 (1988): 2032-2044.

\section{Repository Citation}

Lock, James A., "Cooperative Effects Among Partial Waves in Mie Scattering" (1988). Physics Faculty Publications. 23.

https://engagedscholarship.csuohio.edu/sciphysics_facpub/23

This Article is brought to you for free and open access by the Physics Department at EngagedScholarship@CSU. It has been accepted for inclusion in Physics Faculty Publications by an authorized administrator of EngagedScholarship@CSU. For more information, please contact library.es@csuohio.edu. 


\title{
Cooperative effects among partial waves in Mie scattering
}

\author{
James A. Lock \\ Department of Physics, Cleveland State University, Cleveland, Ohio 44115
}

Received March 28, 1988; accepted July 19, 1988

\begin{abstract}
Observations of an illuminated water droplet at a close distance are described mathematically by the Fourier transform of the Mie-scattering amplitude convolved with the aperture function of the observer's eye. Most of the sharp enhancements found in the Fourier transform correspond to geometrical rays associated with the various terms in the Debye-series expansion of the Mie amplitude. However, there are some enhancements that cannot be ascribed to any individual Debye-series term. Instead, they arise from a constructive interference cooperation of the phase of a scattering resonance in a single partial wave with the region of the stationary phase corresponding to geometrical orbiting in the $m$-internal-reflection portion of the Fourier transform of the scattering amplitude. This phase cooperation amplifies the contribution that the scattering resonance makes to the Fourier-transform ampli-
tude.
\end{abstract}

\section{INTRODUCTION}

The theory of the rainbow has been formulated at many levels of sophistication. In the geometrical-optics theory of Descartes, a rainbow occurs when the angle of the light rays emerging from a water droplet after a number of internal reflections reaches an extremum. ${ }^{1}$ In Airy's wave-optics theory, the distortion of the wave front of the incident light produced by the internal reflections describes the production of the supernumerary bows and predicts a shift of a few tenths of a degree in the angular position of the rainbow from its geometrical-optics location. ${ }^{2}$ In Mie theory, the rainbow appears as a strong enhancement in the electric field scattered by the water droplet. ${ }^{3}$ Although the Mie electric field is the exact solution to the light-scattering problem, it takes the form of an infinite series of partialwave contributions that is slowly convergent and whose terms have a mathematically complicated form. ${ }^{4}$ In the complex angular momentum theory, the sum over partial waves is replaced by an integral, and the rainbow appears as a confluence of saddle-point contributions in the portion of the integral that describes light rays that have undergone $m$ internal reflections within the water droplet. ${ }^{5}$

The rainbows seen in the sky are produced by a large number of water droplets. When an individual water droplet is observed at a close distance (as defined in Section 3), a number of colored glare spots are seen on its surface. ${ }^{6}$ These colored glare spots are produced by the eye of the observer focusing the light rays that emerge from the droplet and that would have otherwise continued on their paths and contributed to the atmospheric rainbow seen at far distances (as defined in Section 3). Mathematically, these glare spots are sharp spikes in the Fourier transform of the Mie electric field convolved with the aperture of the pupil of the observer's eye. ${ }^{7}$ When the Fourier-transform spikes were compared with the predictions of the various-order internalreflection terms of the Debye expansion ${ }^{8}$ of the Mie electric field, it was found that some of the spikes appearing at the edge of the droplet seemed not to correspond to any individual Debye-expansion term. These spikes did not appear to be produced by surface waves that generate light rays that are shed tangentially from the droplet and provide a small background intensity at the edges of the droplet for all observation angles. The additional sharp spikes observed in Ref. 7 represent instead an enhancement over and above this background.

The purpose of this paper is to examine in detail the behavior of these additional Fourier-transform spikes and to demonstrate that they are a cooperative effect. Mathematically, the point of view that we take is as follows. The scattering amplitude and its Fourier transform may be written as double sums. One sum is over partial waves $l$ in the expansion of the incoming and outgoing fields. The other sum is over the number of internal reflections $m$ that a given partial wave undergoes before exiting the droplet. The usual analyses of Mie scattering find interesting structure in the scattering amplitude, namely, geometrical rays and the various-order rainbows, when the $l$ sum is obtained for a fixed value of $m$. The narrow spikes in the Fourier transform of the scattering amplitude obtained by such analyses are termed Debye glare spots, since each of them corresponds to a definite number of internal reflections and a definite term in the Debye expansion. In this paper it is shown that the interesting structure in the Fourier transform of the scattering amplitude is also found when the $m$ sum is obtained for a fixed value of $l$. The scattering enhancements that are produced in this way are termed non-Debye enhancements, since they do not correspond to a definite number of internal reflections. By comparing the two methods for evaluating the scattering-amplitude double sum, it is shown that obtaining the $l$ sum for fixed $m$ emphasizes certain geometrical-raylike aspects of Mie scattering and that obtaining the $m$ sum for fixed $l$ emphasizes certain wavelike aspects of Mie scattering.

Physically, the analysis of the non-Debye enhancements ties together many diverse aspects of Mie scattering. At certain observation angles, geometrical rays making large numbers of internal reflections within the droplet produce glare spots at the right- and left-hand edges of the droplet. They provide a background intensity there whose magnitude is independent of the size of the droplet. Associated with these glare spots are additional geometrical rays be- 
yond the edge of the droplet that are captured by it and that resemble orbiting rays. At certain droplet sizes, scattering resonances occur in individual partial waves. Their constructive interference with the orbiting rays produces the large non-Debye enhancements in the Fourier transform of the scattering amplitude that were reported in Ref. 7. At the same time, a scattering resonance generates a family of internal-reflection resonances. Each member of this family is a constructive interference cooperation of the internalreflection terms of the Debye series for a single partial wave. The scattering resonance constructively interferes with all the members of the internal-reflection-resonance family and additionally strengthens the non-Debye peaks.

\section{DEBYE-SERIES EXPANSION OF THE SCATTERING AMPLITUDE}

The interaction of a plane electromagnetic wave whose wave vector is

$$
k=\frac{2 \pi}{\lambda}
$$

and whose electric field is

$$
\mathbf{E}_{\text {incident }}(\mathbf{r})=E_{0} \exp [i(k z-\omega t)] \hat{u}_{x}
$$

with a dielectric sphere whose radius is $a$ and whose index of refraction is $n$ produces an outgoing scattered electromagnetic wave $\mathbf{E}_{\text {scattered }}(\mathbf{r})$. The amplitude of the scattered wave is found by a partial-wave analysis in which the incident wave is expanded in terms of spherical-multipole standing waves and the scattered wave is expanded in terms of radially outgoing spherical-multipole waves. The farfield limit of the partial-wave-expanded scattered wave is ${ }^{9}$

$$
\begin{aligned}
\lim _{r \rightarrow \infty} \mathbf{E}_{\text {scattered }}(\mathbf{r})= & \frac{i E_{0}}{k r} \exp [i(k r-\omega t)] \\
& \times\left[S_{1}(\theta) \sin \phi \hat{u}_{\phi}-S_{2}(\theta) \cos \phi \hat{u}_{\theta}\right],
\end{aligned}
$$

where $\theta$ and $\phi$ are the angles of the spherical coordinate system. The scattering functions $S_{1}(\theta)$ and $S_{2}(\theta)$ are given by

$$
\begin{aligned}
& S_{1}(\theta)=\sum_{l=1}^{\infty} \frac{2 l+1}{l(l+1)}\left[a_{l} \pi_{l}(\theta)+b_{l} \tau_{l}(\theta)\right], \\
& S_{2}(\theta)=\sum_{l=1}^{\infty} \frac{2 l+1}{l(l+1)}\left[a_{l} \tau_{l}(\theta)+b_{l} \pi_{l}(\theta)\right],
\end{aligned}
$$

and their angular dependences are

$$
\pi_{l}(\theta)=\frac{1}{\sin \theta} P_{l}^{1}(\cos \theta)
$$

and

$$
\tau_{l}(\theta)=\frac{\mathrm{d}}{\mathrm{d} \theta} P_{l}^{\mathrm{I}}(\cos \theta)
$$

The partial-wave scattering amplitudes $a_{l}$ and $b_{l}$ are expressed in terms of the Riccati-Bessel functions

$$
\begin{gathered}
\partial_{l}(w)=w j_{l}(w), \\
\mathcal{N}_{l}(w)=w n_{l}(w),
\end{gathered}
$$

where $j_{l}(w)$ and $n_{l}(w)$ are the spherical Bessel and Neumann functions, respectively. If we define

$$
x \equiv k a, \quad y \equiv n k a
$$

and let

$$
\begin{aligned}
& t_{l}^{1}=\partial_{l}(x) \partial_{l}^{\prime}(y)-n \mathscr{\alpha}_{l}^{\prime}(x) \partial_{l}(y), \\
& t_{l}^{2}=\mathcal{N}_{l}(x) \mathcal{N}_{l}^{\prime}(y)-n \mathcal{N}_{l}^{\prime}(x) \mathcal{N}_{l}(y), \\
& t_{l}^{3}=\mathcal{N}_{l}(x) \delta_{l}^{\prime}(y)-n \mathcal{N}_{l}^{\prime}(x) \alpha_{l}(y) \text {, } \\
& t_{l}^{4}=\partial_{l}(x) \mathcal{N}_{l}^{\prime}(y)-n \partial_{l}^{\prime}(x) \mathcal{N}_{l}(y) \text {, }
\end{aligned}
$$

then $a_{l}$ is given by

$$
a_{l}=\frac{t_{l}^{1}}{t_{l}^{1}+i t_{l}^{3}}
$$

On the other hand, if we let

$$
\begin{aligned}
t_{l}^{1} & =n \partial_{l}(x) \partial_{l}^{\prime}(y)-\partial_{l}^{\prime}(x) \partial_{l}(y), \\
t_{l}^{2} & =n \mathcal{N}_{l}(x) \mathcal{N}_{l}^{\prime}(y)-\mathcal{N}_{l}^{\prime}(x) \mathcal{N}_{l}(y), \\
t_{l}^{3} & =n \mathcal{N}_{l}(x){\partial_{l}^{\prime}}_{l}(y)-\mathcal{N}_{l}^{\prime}(x) \partial_{l}(y), \\
t_{l}^{4} & =n \partial_{l}(x) \mathcal{N}_{l}^{\prime}(y)-{\partial_{l}^{\prime}}_{l}^{\prime}(x) \mathcal{N}_{l}(y),
\end{aligned}
$$

then $b_{l}$ is given by

$$
b_{l}=\frac{t_{l}^{1}}{t_{l}^{1}+i t_{l}^{3}}
$$

The spherical-multipole standing waves in the partialwave expansion of the incident plane wave may be written as linear combinations of radially incoming and outgoing spherical-multiple waves. It is of interest to determine the fractions of each of these multipole amplitudes that are reflected and transmitted when the radially incoming and outgoing waves hit the droplet surface. Region 1 is taken to be the interior of the droplet, and region 2 is taken to be outside the droplet. The reflection and transmission coefficients for the radially incoming and outgoing multipole waves are found to be ${ }^{8}$

$$
\begin{aligned}
& R_{l}^{11}=\frac{-\left(t_{l}^{1}-t_{l}^{2}\right)-i\left(t_{l}^{3}+t_{l}^{4}\right)}{\left(t_{l}^{1}+t_{l}^{2}\right)+i\left(t_{l}^{3}-t_{l}^{4}\right)}, \\
& R_{l}^{22}=\frac{-\left(t_{l}^{1}-t_{l}^{2}\right)+i\left(t_{l}^{3}+t_{l}^{4}\right)}{\left(t_{l}^{1}+t_{l}^{2}\right)+i\left(t_{l}^{3}-t_{l}^{4}\right)}, \\
& T_{l}^{21}=\frac{-2 i / x^{2}}{\left(t_{l}^{1}+t_{l}^{2}\right)+i\left(t_{l}^{3}-t_{l}^{4}\right)}, \\
& T_{l}^{12}=\frac{-2 i / n x^{2}}{\left(t_{l}^{1}+t_{l}^{2}\right)+i\left(t_{l}^{3}-t_{l}^{4}\right)} .
\end{aligned}
$$

In these expressions, the first and second superscripts denote the regions that the spherical-multipole wave is in before and after the interaction with the droplet surface, respectively.

The partial-wave scattering amplitudes may be written as an infinite series of interactions of the spherical-multipole waves with the droplet surface. ${ }^{9}$ Specifically, it is found that 


$$
\frac{t_{l}^{1}}{t_{l}^{1}+i t_{l}^{3}}=\frac{1}{2}\left(1-R_{l}^{22}-\frac{T_{l}^{21} T_{l}^{12}}{1-R_{l}^{11}}\right),
$$

or, when the last term is written as a geometric series,

$$
\frac{t_{l}^{1}}{t_{l}^{1}+i t_{l}^{3}}=\frac{1}{2}\left[1-R_{l}^{22}-\sum_{m=0}^{\infty} T_{l}^{21}\left(R_{l}^{11}\right)^{m} T_{l}^{12}\right] .
$$

Equation (2.20) is known as the Debye-series expansion of the partial-wave scattering amplitudes. The various terms of this series have simple physical interpretations. The first term, when summed over $l$, describes the diffraction of light around the droplet. ${ }^{10}$ The second term represents the outgoing spherical-multipole waves that have reflected from the surface of the droplet. We shall call this the direct-reflection term. The geometric series describes the light that penetrates the droplet. The $m=0$ term of the sum represents the outgoing waves that were transmitted into the droplet, propagated radially inward to the center and passed through it, propagated radially outward, and then transmitted through the surface. The $m \geq 1$ terms represent the outgoing waves that propagated repetitively radially inward and outward within the droplet, internally reflecting $m$ times before emerging from it. The entire geometric series is called the internal-reflection sum.

This interpretation is strengthened when the electric field within the spherical droplet is partial-wave expanded into spherical-multipole standing waves. The partial-wave amplitudes inside the droplet are

$$
c_{l}=\frac{-i}{t_{l}^{1}+i t_{l}^{3}}
$$

for Eqs. (2.11) and

$$
d_{l}=\frac{-i}{t_{l}^{1}+i t_{l}^{3}}
$$

for Eqs. (2.13). These amplitudes may be rewritten as

$$
\frac{-i}{t_{l}^{1}+i t_{l}^{3}}=\frac{T_{l}^{21}}{1-R_{l}^{11}}=\sum_{m=0}^{\infty} T_{l}^{21}\left(R_{l}^{11}\right)^{m}
$$

again suggesting multiple internal reflections of spherical multipoles within the droplet. Equations (2.20) and (2.23) are analogous to the treatment of the reflection and transmission of a plane wave interacting with a thin dielectric film. For the plane geometry as well, the total reflected and transmitted waves may be written in terms of infinite series of partial reflections and transmissions at the front and rear surfaces of the film. ${ }^{11,12}$

In the thin-film problem, the magnitude of each complex term in the infinite series for reflection or transmission describes the fraction of the incident wave that has been reflected or transmitted to that point. The phase of each complex term describes the phase delay of the wave that is due to the path that it has traveled within the film to that point. The complex spherical-multipole reflection and transmission coefficients appearing in Eq. (2.20) may also be written in terms of magnitudes and phases as

$$
\begin{aligned}
& R_{l}^{11}=r_{l}^{11} \exp \left(i \phi_{l}^{11}\right), \\
& T_{l}^{21}=n T_{l}^{12}=t_{l}^{21} \exp \left(i \phi_{l}^{21}\right),
\end{aligned}
$$

$$
R_{l}^{22}=r_{l}^{22} \exp \left(i \phi_{l}^{22}\right)
$$

These have a similar physical significance, which is pursued in Section 4. Equations (2.15)-(2.18) determine that $r_{l}^{11}$, $\phi_{l}^{11}$, and $\phi_{l}^{21}$ are the only independent variables in Eqs. (2.24)-(2.26) with

$$
\begin{aligned}
& r_{l}^{22}=r_{l}^{11}, \\
& t_{l}^{21}=\left\{n\left[1-\left(r_{l}^{11}\right)^{2}\right]\right\}^{1 / 2},
\end{aligned}
$$

and

$$
\phi_{l}^{22}=2 \phi_{l}^{21}-\phi_{l}^{11}-\pi
$$

For $l<\infty$, we also have ${ }^{8} r_{l}^{11}<1$. By a combination of Eqs. (2.15), (2.17), (2.24), and (2.25), the independent phases $\phi_{l}^{21}$ and $\phi_{l}^{11}$ may be written as

$$
\phi_{l}^{21}=\arctan \left(\frac{t_{l}^{1}+t_{l}^{2}}{t_{l}^{3}-t_{l}^{4}}\right)
$$

and

$$
\phi_{l}^{11}=\arctan \left[\frac{2\left(t_{l}^{1} t_{l}^{4}+t_{l}^{2} t_{l}^{3}\right)}{\left(t_{l}^{1}\right)^{2}+\left(t_{l}^{3}\right)^{2}-\left(t_{l}^{2}\right)^{2}-\left(t_{l}^{4}\right)^{2}}\right] .
$$

When these magnitudes and phases are used in Eq. (2.20), the partial-wave scattering amplitudes become

$$
\begin{aligned}
\frac{t_{l}^{1}}{t_{l}^{1}+i t_{l}^{3}}= & \frac{1}{2}\left\{1+r_{l}^{11} \exp \left[i\left(2 \phi_{l}^{21}-\phi_{l}^{11}\right)\right]\right. \\
& -\sum_{m=0}^{\infty}\left[1-\left(r_{l}^{11}\right)^{2}\right]\left(r_{l}^{11}\right)^{m} \\
& \left.\times \exp \left[i\left(2 \phi_{l}^{21}+m \phi_{l}^{11}\right)\right]\right\}
\end{aligned}
$$

In Section 4 the phases $\phi_{l}^{21}$ and $\phi_{l}^{11}$ are evaluated in the geometrical-optics limit.

From numerical computations it was found that the partial-wave series may be cut off at

$$
l_{\max } \approx x+4.3 x^{1 / 3}
$$

with no loss of accuracy. ${ }^{13}$ Physically this corresponds to the size of the region outside the water droplet for which incident light rays can be deflected appreciably by it. The interval

$$
x-4.3 x^{1 / 3} \leq l \leq x+4.3 x^{1 / 3}
$$

is called the edge region for the incident partial waves.

\section{FOURIER TRANSFORM OF THE SCATTERING FUNCTIONS}

As in Ref. 7, we shall model the electric field on the retina of an observer viewing a single water droplet at a close distance by the Fourier transform of the Mie scattering amplitude convolved with the observer's pupil-aperture function. A close distance is defined as a distance far enough away from the droplet that far-field Mie scattering is used but close enough that the droplet subtends an angle of a few degrees at the position of the observer. Specifically, we consider 


$$
F_{i}\left(p, \theta_{0}\right)=\int_{-\theta_{u} / 2}^{\theta_{u} / 2} \mathrm{~d} \xi S_{i}\left(\theta_{0}+\xi\right) e^{-i p \xi},
$$

where $S_{i}$ are the Mie-scattering functions of Eqs. (2.4) and (2.5), $\theta_{0}$ is the observation angle, $\theta_{w}$ is the angular width of the pupil aperture, and $p$ is the spatial-frequency variable of the Fourier transform $F_{i}$. The spatial frequency $p$ has two interpretations. Mathematically it is the number of radians of oscillation of the scattering function $S_{i}$ per degree of scattering angle. For example, classically the largest partial wave $l$ contributing to $S_{i}$ is associated by means of the localization principle with the light ray that grazes the edge of the sphere. ${ }^{14,15}$ This occurs for $l=x$. The angular dependence of this partial-wave contribution has $x$ cycles or $2 \pi x \operatorname{rad}$ of oscillation per $360 \mathrm{deg}$ of its argument, giving the spatial frequency $p=x$. In its second interpretation, $p$ visually corresponds to a position on a cross section of the sphere, with $p=0$ being the center of the sphere and $p= \pm x$ being the right- and left-hand edges.

When Eqs. (2.4) and (2.5) are substituted into Eq. (3.1), the Fourier transform of the Mie-scattering functions convolved with the pupil aperture becomes

$$
\begin{aligned}
& F_{1}\left(p, \theta_{0}\right)=\sum_{l=1}^{l_{\max }} \frac{2 l+1}{l(l+1)}\left[a_{l} U_{l}\left(p, \theta_{0}\right)+b_{l} V_{l}\left(p, \theta_{0}\right)\right], \\
& F_{2}\left(p, \theta_{0}\right)=\sum_{l=1}^{l_{\max }} \frac{2 l+1}{l(l+1)}\left[a_{l} V_{l}\left(p, \theta_{0}\right)+b_{l} U_{l}\left(p, \theta_{0}\right)\right],
\end{aligned}
$$

where

$$
U_{l}\left(p, \theta_{0}\right)=\int_{-\theta_{\mu} / 2}^{\theta_{u} / 2} \mathrm{~d} \xi \pi_{l}\left(\theta_{0}+\xi\right) e^{-i p \xi}
$$

and

$$
V_{l}\left(p, \theta_{0}\right)=\int_{-\theta_{w} / 2}^{\theta_{w} / 2} \mathrm{~d} \xi \tau_{l}\left(\theta_{0}+\xi\right) e^{-i p \xi} .
$$

We are interested primarily in partial waves in the edge region for large water droplets with $x \gg 1$. In this region and for $\theta_{0}$ far from the forward and backward directions, $\pi_{l}(\theta)$ and $\tau_{l}(\theta)$ may be approximated by their asymptotic forms, ${ }^{16}$ and we have

$$
\begin{aligned}
V_{l}\left(p, \theta_{0}\right) \approx & \frac{\theta_{w}}{2}\left(\frac{2 l^{3}}{\pi}\right)^{1 / 2} \exp (-i \pi / 4)\left\{\exp \left(i l \theta_{0}\right) \operatorname{sinc}\left[(p-l) \frac{\theta_{w}}{2}\right]\right. \\
& \left.+\exp \left(-i l \theta_{0}\right) \operatorname{sinc}\left[(p+l) \frac{\theta_{w}}{2}\right]\right\} \\
U_{l}\left(p, \theta_{0}\right) \approx & \frac{1}{l} V_{l}\left(p, \theta_{0}\right)
\end{aligned}
$$

where

$$
\operatorname{sinc}(w)=\frac{\sin w}{w} .
$$

The sinc function peaks at $w=0$ and falls to half its peak height at $w \approx \pm 1.89$. When relations (3.6) and (3.7) are substituted into Eqs. (3.2) and (3.3), the Fourier transform of the scattering functions acquires a phase that depends on the observation angle and

$$
\begin{aligned}
F_{1}\left(p, \theta_{0}\right) \approx & \frac{\theta_{w}}{2}\left(\frac{2}{\pi}\right)^{1 / 2} \exp (-i \pi / 4) \sum_{l=1}^{l_{\max }} \frac{2 l+1}{l(l+1)} l^{3 / 2} b_{l} \\
& \times\left\{\exp \left(i l \theta_{0}\right) \operatorname{sinc}\left[(p-l) \frac{\theta_{w}}{2}\right]\right. \\
& \left.+\exp \left(-i l \theta_{0}\right) \operatorname{sinc}\left[(p+l) \frac{\theta_{w}}{2}\right]\right\}, \\
F_{2}\left(p, \theta_{0}\right) \approx & \frac{\theta_{w}}{2}\left(\frac{2}{\pi}\right)^{1 / 2} \exp (-i \pi / 4) \sum_{l=1}^{l_{\max }} \frac{2 l+1}{l(l+1)} l^{3 / 2} a_{l} \\
& \times\left\{\exp \left(i l \theta_{0}\right) \operatorname{sinc}\left[(p-l) \frac{\theta_{w}}{2}\right]\right. \\
& \left.+\exp \left(-i l \theta_{0}\right) \operatorname{sinc}\left[(p+l) \frac{\theta_{w}}{2}\right]\right\} .
\end{aligned}
$$

When all the constants and slowly varying functions of $l$ are combined into the quantity $K$, these Fourier transforms may be written collectively as

$$
\begin{aligned}
F_{i}\left(p, \theta_{0}\right) \approx & K \sum_{l=1}^{l_{\max }} \frac{1}{2}\left\{1+r_{l}^{11} \exp \left[i\left(2 \phi_{l}^{21}-\phi_{l}^{11}\right)\right]\right. \\
& \left.-\sum_{m=0}^{\infty}\left[1-\left(r_{l}^{11}\right)^{2}\right]\left(r_{l}^{11}\right)^{m} \exp \left[i\left(2 \phi_{l}^{21}+m \phi_{l}^{11}\right)\right]\right\} \\
& \times\left\{\exp \left(i l \theta_{0}\right) \operatorname{sinc}\left[(p-l) \frac{\theta_{w}}{2}\right]+\exp \left(-i l \theta_{0}\right)\right. \\
& \left.\times \operatorname{sinc}\left[(p+l) \frac{\theta_{w}}{2}\right]\right\} .
\end{aligned}
$$

The constructive interference of the overlapping sinc functions of a number of adjacent or nearly adjacent $l$ values produces the glare spots that the observer sees on the surface of the droplet. The presence of the observer is instrumental in the production of the glare spots, since his or her aperture function determines the degree of overlap of the sinc functions. For a wide aperture, the scattering functions are sampled over a large angular interval, the angular dependences $\tau_{l}(\theta)$ and $\pi_{l}(\theta)$ are determined accurately, the sinc functions approach $\delta$ functions, and there is virtually no overlap between the sinc functions of different partial waves. No cooperative effects based on the overlap and the coherent interference of the sinc functions can occur. For a small aperture width, the scattering functions are sampled over a smaller interval, $\tau_{l}(\theta)$ and $\pi_{l}(\theta)$ are determined less accurately, the sinc functions are broader functions of $l$, and substantial overlap between the sinc functions of different partial waves occurs. However, if $\theta_{w}$ becomes too small and the sinc functions become too broad, then all the partialwave contributions overlap, and the observer sees merely the Mie-scattered intensity at his or her observation angle and not a blurred version of its Fourier transform. Thus the cooperative effects caused by the overlap of the sinc functions of a limited number of partial waves are produced only when $\theta_{w}$ is in the intermediate region, of the order of a few degrees. For

$$
\theta_{w}=4.6^{0}
$$


the sinc functions of approximately 50 partial waves to either side of the central partial wave overlap substantially.

\section{GEOMETRICAL-RAY GLARE SPOTS}

The customary way to evaluate the interaction portion of the scattering amplitude is to convert the $l$ sum to an integral for each value of $m$ by using the modified Watson transformation and then to evaluate and interpret the principal contributions to the integrals. The saddle-point or stationarypoint contributions to the integrals are reminiscent of Fermat's principle and correspond to geometrical rays. If the $l$ sum is obtained for fixed $m$ without being converted into an integral, geometrical rays are produced when a number of adjacent partial waves in the $l$ sum occur with the same phase and constructively interfere. ${ }^{17}$ The path of such a stationary-point geometrical ray through the droplet and beyond it is identical to the light path that would be observed if a large spherical globe filled with water were illumi-

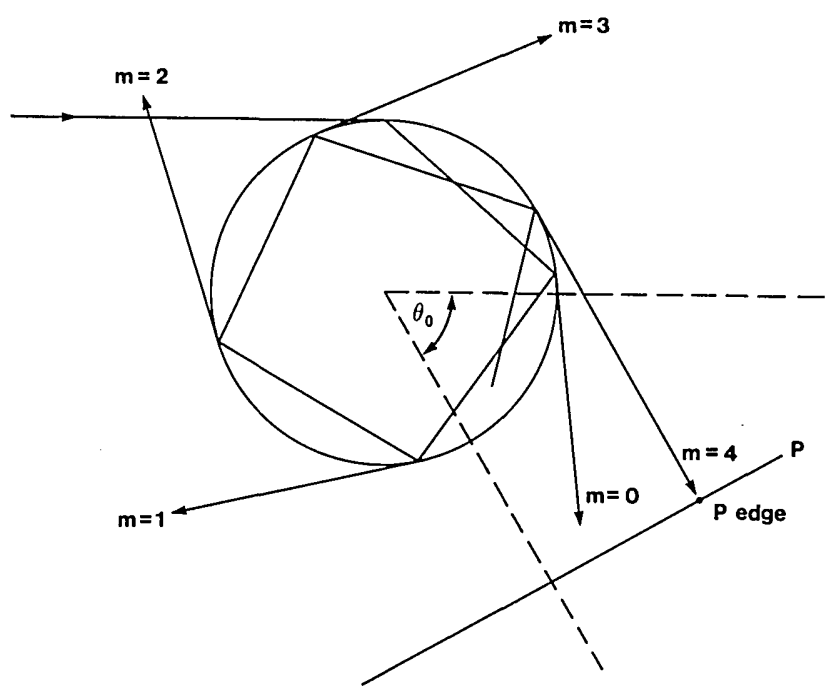

(a)

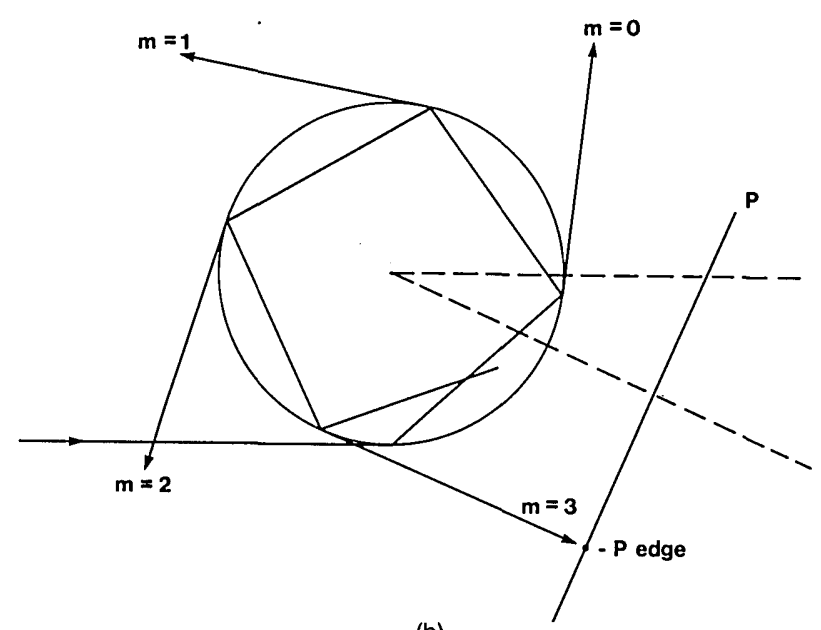

(b)

Fig. 1. (a) Geometrical rays produced by a light ray incident upon the top of the droplet. The exiting rays appear on the right side of the droplet. The observation angle is $\theta_{0}$. (b) Geometrical rays produced by a light ray incident upon the bottom of the droplet. The exiting rays appear on the left side of the droplet. nated by a small-diameter laser beam. The paths of such geometrical rays are shown in Figs. 1(a) and 1(b). When a ray is incident upon the top half of the droplet, its exit always occurs on the right-hand side of the droplet, corresponding to the sinc function in relation (3.11) that peaks at $p=l$. If a ray is incident upon the bottom half of the droplet, its exit occurs on the left-hand side, corresponding to the sinc function in relation (3.11) that peaks at $p=-l$.

For an observer at the angle $\theta_{0}$ in Fig. 1(a), a geometrical ray making $m$ internal reflections within the droplet and having the impact parameter associated with the incident partial waves centered about the value $l_{0}$ produces a glare spot at the location $p$ on the droplet where ${ }^{7}$

$$
\theta_{l_{0}}^{\text {deviation }}=m \pi+2 \arcsin \left(\frac{p}{x}\right)-(2 m+2) \arcsin \left(\frac{p}{n x}\right)
$$

and

$$
\theta_{0}=\left\{\begin{array}{ll}
\theta_{l_{0}}^{\text {deviation }}[\bmod 2 \pi] & \text { if } 0 \leq \theta_{l_{0}}^{\text {deviation }}[\bmod 2 \pi] \leq \pi \\
-\theta_{l_{0}}^{\text {deviation }}[\bmod 2 \pi] & \text { if }-\pi<\theta_{l_{1}}^{\text {deviation }}[\bmod 2 \pi]<0
\end{array},\right.
$$

where

$$
p=l_{0}
$$

if the ray is incident upon the upper half of the droplet and where

$$
p=-l_{0}
$$

if it is incident upon the lower half. This prediction of the locations of geometrical-ray glare spots allows us to evaluate the phases $\phi_{l}^{21}$ and $\phi_{l}^{11}$ approximately. In the region near the partial wave $l_{0}$, the portion of the Fourier transform of the scattering functions corresponding to $m$ internal reflections may be written as

$$
\begin{aligned}
F_{i}^{(m)}\left(p, \theta_{0}\right) \approx & -\frac{K}{2} \sum_{l=1}^{l_{\text {minx }}}\left[1-\left(r_{l}^{11}\right)^{2}\right]\left(r_{l}^{11}\right)^{m} \exp \left[i\left(2 \phi_{l_{0}}^{21}+m \phi_{l_{0}}^{11}\right)\right] \\
& \times \exp \left[i z\left(2 \Delta \phi_{l_{1}}^{21}+m \Delta \phi_{l_{l}}^{11}\right)\right]\left\{\exp \left(i l_{0} \theta_{0}\right) \exp \left(i z \theta_{0}\right)\right. \\
& \times \operatorname{sinc}\left[\left(p-l_{0}-z\right) \frac{\theta_{w}}{2}\right]+\exp \left(-i l_{0} \theta_{0}\right) \exp \left(-i z \theta_{0}\right) \\
& \left.\times \operatorname{sinc}\left[\left(p+l_{0}+z\right) \frac{\theta_{w}}{2}\right]\right\}
\end{aligned}
$$

where the transmission and reflection phase angles are Taylor-series expanded about $l_{0}$ as

$$
\phi_{l}^{21}=\phi_{l_{1}}^{21}+z \Delta \phi_{l_{l}}^{21}+\ldots
$$

and

$$
\phi_{l}^{11}=\phi_{l_{11}}^{11}=z \Delta \phi_{l_{11}}^{11}+\ldots
$$

with

$$
z=l-l_{0}
$$

According to Fermat's principle, a geometrical ray occurs when the phase of the amplitude is stationary or independent of $z$ for a number of partial waves centered about $l_{0}$. 
For rays entering the upper half of the droplet and producing a glare spot at $p=l$, this occurs for the first sinc function in relation (4.5) when

$$
\theta_{0}=-2 \Delta \phi_{l_{0}}^{21}-m \Delta \phi_{l_{0}}^{11}
$$

or, with Eq. (4.1), when

$$
\Delta \phi_{l_{0}}^{21}=-\arcsin \left(\frac{l_{0}}{x}\right)+\arcsin \left(\frac{l_{0}}{n x}\right)
$$

and

$$
\Delta \phi_{l_{0}}^{11}=2 \arcsin \left(\frac{l_{0}}{n x}\right)-\pi .
$$

For rays entering the lower half of the droplet and producing a glare spot at $p=-l$, the same result is obtained by using the second sinc function in relation (4.5). The Taylor-series expansions of Eqs. (4.6)-(4.8) may also be used in the portion of the Fourier transform of the scattering functions corresponding to direct reflection. In order that the direct reflection ray be produced by constructive interference of the partial waves centered about

$$
p=-l_{0}
$$

and appear in the $\theta_{0}$ direction as given by

$$
p=-x \cos \left(\theta_{0} / 2\right)
$$

the $z$ independence of the Fourier-transform phase again gives Eqs. (4.10) and (4.11).

When $\Delta \phi_{l}^{21}$ and $\Delta \phi_{l}^{11}$ are integrated, we obtain

$$
\begin{aligned}
\phi_{l}^{21} \approx & \left(n^{2} x^{2}-l^{2}\right)^{1 / 2}-\left(x^{2}-l^{2}\right)^{1 / 2}-l \\
& \times\left[\arcsin \left(\frac{l}{x}\right)-\arcsin \left(\frac{l}{n x}\right)\right], \\
\phi_{l}^{11} \approx & 2\left(n^{2} x^{2}-l^{2}\right)^{1 / 2}-l\left[\pi-2 \arcsin \left(\frac{l}{n x}\right)\right]-\pi
\end{aligned}
$$

as the geometrical-optics approximation to the phases of the spherical-multipole transmission and reflection coefficients. The phases of relations (4.14) and (4.15) have interesting physical interpretations. The localization principle ${ }^{14,15}$ associates the partial wave $l$ with the incident geometrical ray whose impact parameter $b$ is given by

$$
b \approx a(l / x) \text {. }
$$

The phase delay produced by the transmission of this geometrical ray into the droplet is

$$
\left(\phi_{l}^{21}\right)_{\text {geometric }}=\left(n^{2} x^{2}-l^{2}\right)^{1 / 2}-\left(x^{2}-l^{2}\right)^{1 / 2} .
$$

This delay is the first term of relation (4.14). The phase delay produced by the internal reflection of the geometrical ray within the droplet is

$$
\left(\phi_{l}^{11}\right)_{\text {geometric }}=2\left(n^{2} x^{2}-l^{2}\right)^{1 / 2} .
$$

This delay again is the first term of relation (4.15). The remaining terms in relations (4.14) and (4.15) that are proportional to $l$ represent a reduction in the phase delay of the partial wave from that of geometrical rays. This is due to the fact that a single partial wave is a spherical multipole and not a geometrical ray. It has the spherical Besselfunction modulation $j_{l}(n k r)$ rather than the sinusoidal mod- ulation associated with geometrical rays. Inside the droplet $j_{l}(n k r)$ undergoes fewer radial oscillations, owing to its being damped at small $r$ by the centrifugal potential. The decrease in the rate of oscillation increases with increasing $l$ and produces the reduction in phase delay. Finally it should be noted that when relation (4.5) is at the stationaryphase point and produces a geometrical ray by constructive interference, substitution of Eqs. (4.1) and (4.2) and relations (4.14) and (4.15) into relation (4.5) shows that the value of the stationary phase is exactly the phase delay expected for the geometrical ray according to Eqs. (4.17) and (4.18).

As a test of the accuracy of the geometrical-optics approximation of relations (4.14) and (4.15), the exact phases $\phi_{l}^{21}$ and $\phi_{l}^{11}$ of Eqs. (2.30) and (2.31) as well as the geometricaloptics approximation of relations (4.14) and (4.15) were evaluated for

$$
\begin{aligned}
& \lambda=0.6328 \mu \mathrm{m}, \\
& a=750.007 \mu \mathrm{m}
\end{aligned}
$$

and for

$$
n=1.331 \text {. }
$$

Relation (4.14) was found to be almost identical to the exact $\phi_{l}^{21}$ for both the $a_{l}$ and the $b_{l}$ polarizations of Eqs. (2.11) and (2.13) for $l \gtrsim 1$. In the interval $1 \leq l \leq x, \phi_{l}^{21}$ varied smoothly through 205 cycles of phase, and relation (4.14) deviated from $\phi_{l}^{21}$ for both polarizations by only approximately $16 \mathrm{deg}$ at $l \approx x$. For $\phi_{l}^{11}$ the situation was slightly more complicated. The phase for the $b_{l}$ polarization again varied smoothly through 2781 cycles for $1 \leq l \leq x$. Relation (4.15) approximated $\phi_{l}^{11}$ well, being almost identical to it for $l \gtrsim 1$ and deviating from it by $\sim 48 \mathrm{deg}$ at $l \approx x$. The phase for the $a_{l}$ polarization also varied smoothly, except in the immediate vicinity of the Brewster-angle partial wave at $l=5954$, where $\phi_{l}^{11}$ rapidly slowed by $180 \mathrm{deg}$ of phase. Below the Brewsterangle partial wave, relation (4.15) was nearly $180 \mathrm{deg}$ out of phase with $\phi_{l}^{11}$, and above the Brewster-angle partial wave, relation (4.15) was nearly in phase with $\phi_{l}^{11}$, again deviating from it by approximately $48 \mathrm{deg}$ at $l \approx x$. For the $a_{l}$ polarization at the Brewster angle, it was also seen that $r_{l}^{11}$ went to zero, thus producing the well-known strong polarization of atmospheric rainbows. ${ }^{18}$

For the most part, the geometrical-optics approximation of Eqs. (4.10) and (4.11) and relations (4.14) and (4.15) provides an accurate description of the structure of the $m$ internal-reflection portion of relation (3.11). For example, when $m=11$, for Eq. (4.21), and $\theta_{0}<88.91 \mathrm{deg}$, a single geometrical ray $R_{a}^{(11)}$ is produced near the left-hand edge of the droplet, corresponding to the second sinc function in relation (3.11). When the observer is at the scattering angle of the critically refracted ray $\theta_{c}^{(11)}=88.91 \mathrm{deg}$, a second geometrical ray $R_{b}^{(11)}$ appears at $p=-x$. For $88.91^{\circ} \leq \theta_{0} \leq$ $93.11^{\circ}$, the $R_{a}^{(11)}$ and $R_{b}^{(11)}$ geometrical-ray glare spots approach each other and merge to form the 11th-order rainbow at the rainbow angle $\theta_{\text {rainbow }}^{(11)}=93.11 \mathrm{deg}$. The Airy correction predicts that the rainbow is shifted downward to $\theta_{\text {Airy }}^{(11)}=$ $91.22 \mathrm{deg}$, and the actual inflection point in the phase of the Mie 11th-order Debye amplitude occurs at $\theta_{0}=92.6^{\circ}$ for the $a_{l}$ polarization and at $\theta_{0}=92.9 \mathrm{deg}$ for the $b_{l}$ polarization.

In Fig. 2, the phase of the second sinc-function contribution for $m=11$ to $F_{2}\left(p, \theta_{0}=90 \mathrm{deg}\right)$ is plotted as a function 


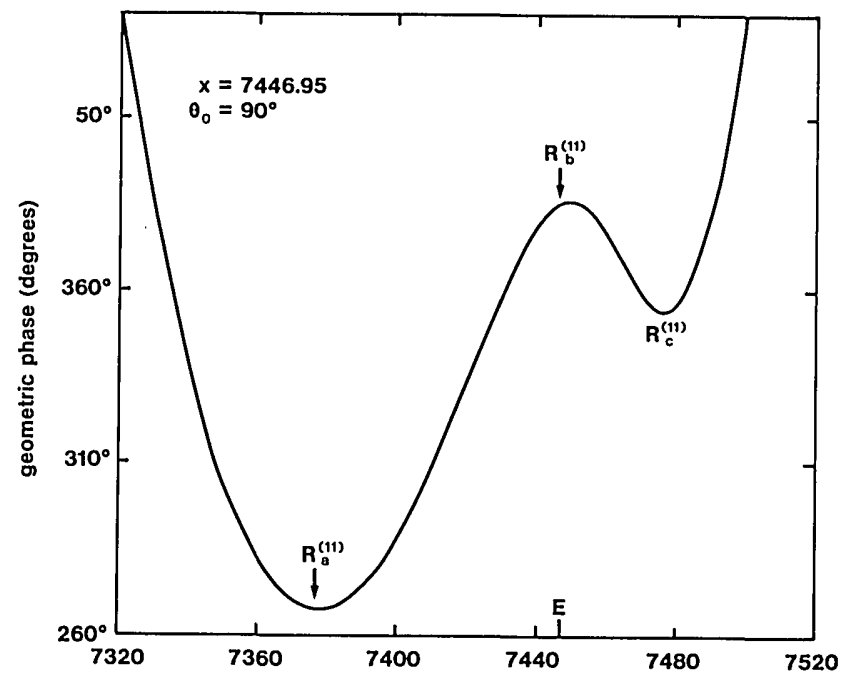

Fig. 2. Phase of the $m=11$ portion of relation (3.11) as a function of the partial wave $l$. The arrows correspond to the locations of the geometrical rays $R_{a}^{(11)}$ and $R_{b}^{(11)}$ according to Eq. (4.1). The stationary-phase point $R_{c}^{(11)}$ corresponds to an orbiting ray. The partial wave labeled $\mathrm{E}$ marks the geometric edge of the droplet.

of $l$. The angles $\phi_{l}^{21}$ and $\phi_{l}^{11}$ contributing to this phase were calculated by using the exact Eqs. (2.30) and (2.31). The two points of stationary phase labeled $R_{a}^{(11)}$ and $R_{b}^{(11)}$ correspond to the two geometrical rays described above. The geometrical-optics locations of the two rays predicted by Eqs. (4.1) and (4.2) are denoted by the arrows in Fig. 2. In addition to these, there is a third point of stationary phase, labeled by $R_{c}^{(11)}$ in Fig. 2, occurring beyond the geometrical edge of the droplet. This point of stationary phase has the following behavior. At $\theta_{c}^{(11)}$, the $m=11$ phase has an inflection point for $l$ slightly larger than $x$. In terms of partial waves, this corresponds to the spherical-multipole standing waves that are just below the top of the centrifugal barrier and are able to tunnel through it and to interact with the droplet. $8,19,20$ Alternatively, it corresponds to a geometrical ray with a small but finite width or profile that is incident just beyond the edge of the droplet. A small amount of amplitude at the edge of the ray profile overlaps the edge of the droplet and is captured by it, being transmitted inside with the critical angle of refraction and then multiply internally reflecting. For $\theta_{0}>\theta_{c}^{(11)}$, the inflection point splits into a relative maximum, $R_{b}^{(11)}$, which corresponds to a geometrical ray and moves downward in $l$ as $\theta_{0}$ is increased, and a relative minimum, $R_{c}^{(11)}$, which moves upward in $l$ farther beyond the edge of the droplet as $\theta_{0}$ is increased. The $R_{c}^{(11)}$ stationary point may again be interpreted in two complementary ways. First, it corresponds to spherical multipoles farther below the top of the centrifugal barrier and whose interaction with the droplet by means of barrier penetration is decreased. Second, it corresponds to an incident geometrical ray increasingly farther beyond the edge of the droplet and having a decreasing amount of its amplitude at the outer edge of the ray profile being captured by the droplet. As the $l$ for which $R_{c}^{(11)}$ occurs increases beyond $x$, the deflection angle of the ray decreases, since the droplet cannot interact with it so strongly. Similarly, the strength of the deflected ray rapidly decreases, since

$$
\lim _{l \gg x}\left[1-\left(r_{l}^{11}\right)^{2}\right]\left(r_{l}^{11}\right)^{11}=0 .
$$

This effect is reminiscent of the semiclassical phenomenon of orbiting, ${ }^{19}$ and $R_{c}^{(11)}$ is called the orbiting-ray stationaryphase point. Such a stationary-phase point occurs in the phase of the Fourier transform of every term of the Debye series, including the $m=0$ transmission term. The $R_{c}^{(m)}$ stationary-phase point is more pronounced and occurs over a larger range of $\theta_{0}$ for increasing $m$. This effect is not associated with surface waves, since surface waves occur most strongly on the shadow side of the scattering angle of the critically refracted ray and the orbiting rays occur only on the lit side. In Section 7 it is shown that scattering resonances cooperate with the orbiting-ray stationary-phase point to produce the non-Debye enhancements in the Fourier transform of the scattering functions.

\section{INTERNAL-REFLECTION RESONANCES IN THE EDGE REGION}

As mentioned at the beginning of Section 4, the usual way to evaluate the scattering amplitude is to obtain the $l$ sum for a fixed value of $m$ and then to examine the most-significant $m$ values for the scattering angle of interest. We now consider the opposite point of view and evaluate the $m$ sum for a fixed value of $l$. The reason for doing this is suggested by relation (4.5). Near the edge of the droplet, where $r_{l}^{11}$ is near unity, all the Debye-series terms in a partial-wave scattering amplitude have small and nearly identical magnitudes but different phases. Thus, once inside the droplet, a spherical multipole can reflect back and forth within it many times with a large amplitude. This causes the Debye series to be slowly convergent. If the phases of the different internalreflection terms in the sum constructively interfere by having

$$
\phi_{l}^{11}=0,
$$

the entire sum is quite large even though each of the individual contributions to it is small. Specifically, we may write

$$
\sum_{m=0}^{\infty} T_{l}^{21}\left(R_{l}^{11}\right)^{m} T_{l}^{12}=\frac{\left[1-\left(r_{l}^{11}\right)^{2}\right] \exp \left[i\left(2 \phi_{l}^{21}+\gamma_{l}\right)\right]}{\left[\left(1-r_{l}^{11}\right)^{2}-4 r_{l}^{11} \sin ^{2}\left(\phi_{l}^{11} / 2\right)\right]^{1 / 2}},
$$

where

$$
\tan \gamma_{l}=\frac{r_{l}^{11} \sin \phi_{l}^{11}}{1-r_{l}^{11} \cos \phi_{l}^{11}}
$$

The magnitude of the internal-reflection sum of Eq. (5.2) reaches its maximum value when Eq. (5.1) is satisfied. The right-hand side of Eq. (5.2), when considered as a function of $\phi_{l}^{11}$, has the form of a resonant amplitude whose full width at half-maximum is $\delta_{l}^{11}$, where

$$
\sin \left(\frac{\delta_{l}^{11}}{4}\right)=\frac{\left(1-r_{l}^{11}\right)}{2}\left(\frac{3}{r_{l}^{11}}\right)^{1 / 2} .
$$

Thus we call the condition of Eq. (5.1) an internal-reflection resonance. Physically it corresponds to the partial-wave spherical multipole $l$ traveling an integer number of wavelengths within the droplet between successive internal reflections. The partially transmitted wave at each of the 
successive surface interactions then has the same phase as all the others and constructively interferes with them as they propagate radially outward toward the observer.

Under most circumstances, internal-reflection resonances produce no observable effects in the scattering functions of Eqs. (2.4) and (2.5). This can be seen in the following way. At an internal-reflection resonance, the internal-reflection sum in Eq. (2.20) attains the value

$$
-\left(1+r_{l}^{11}\right) \exp \left(2 i \phi_{l}^{21}\right) \text {. }
$$

However, the direct-reflection term becomes

$$
r_{l}^{11} \exp \left(2 i \phi_{l}^{21}\right)
$$

and cancels the internal reflection enhancement unless $\phi_{l}^{21}$ is $90 \mathrm{deg}$ as well. For this exception, which corresponds to a scattering resonance, as is shown in Section 6, the diffractive amplitude nearly cancels the direct-reflection amplitude instead and preserves the internal-reflection enhancement. This usual cancellation of the internal-reflection sum by the direct-reflection term in the partial-wave scattering amplitude under the condition of an internal-reflection resonance is analogous to the case of the total-reflection amplitude for the thin-film example when the film thickness is $N / 2$ wavelengths. For this thickness, each term of the multiple-internal-reflection sum has the same phase and exhibits constructive interference in the reflected direction. However, this constructive interference sum is $180 \mathrm{deg}$ out of phase with the direct reflection, giving a net destructive interference for reflection. This cancellation does not occur in the Fourier transform of the scattering functions and results in the displacement of the direct-reflection glare spot from the internal-reflection glare spot.

\section{CONNECTION BETWEEN SCATTERING RESONANCES AND INTERNAL-REFLECTION RESONANCES}

A scattering resonance occurs in the partial wave $l$ at the size parameter $x$ when

$$
t_{l}^{3}=0
$$

in Eqs. (2.11) or (2.13). Physically, a scattering resonance corresponds to a natural mode of electromagnetic vibration of the droplet. ${ }^{4}$ At resonance the droplet captures an anomalously large amount of energy from the initial partial wave $l$, stores it within the droplet in the form of the $l$ multipole standing wave, ${ }^{21,22}$ and later releases it in the form of a largeamplitude outgoing $l$ multipole spherical wave. This largeamplitude outgoing wave is responsible for the narrow enhancements observed in the total-scattering cross section ${ }^{23-}$ ${ }^{25}$ and in the glory-region intensity ${ }^{17,26}$ when they are plotted as functions of the size parameter $x$.

In this section it is shown that, for resonances at the upper end of the edge region of relation (2.34), a resonant spherical multipole corresponds to an internal-reflection resonance. This mechanism accounts for all the observed properties of scattering resonances. Since $r_{l}^{11}$ is near unity for a resonant partial wave, the portion of the spherical multipole that enters the droplet reflects back and forth within it many times, producing a long path within the droplet. This long path length makes the resonance susceptible to damping by the presence of an imaginary component of the index of refraction. Since the phase delay between successive internal reflections is an integer number of cycles at an internalreflection resonance, the portion of the amplitude that is transmitted outside at each surface interaction constructively interferes with every other one, producing a strong scattered wave. The fact that there are many such internalreflection surface interactions gives rise to the delay of the scattered wave with respect to the prompt direct reflection. ${ }^{27,28}$ It should be remembered that a scattering resonance in the single partial wave $l_{r}$ corresponds to a radial motion of the spherical multipole within the droplet. It is not to be confused with the separate phenomenon of geometrical resonances described in Refs. 29 and 30. Geometrical resonances constitute a special class of light rays that multiply internally reflect within the droplet. Being light rays, geometrical resonances are not made from a single partial wave. They are made from the constructive interference of many adjacent partial waves.

In general, scattering resonances and internal-reflection resonances are distinct from each other, since a scattering resonance occurs when

$$
\phi_{l}^{11}=\phi_{l}^{21} \pm \arccos \left(\frac{\cos \phi_{l}^{21}}{r_{l}^{11}}\right)
$$

and an internal-reflection resonance occurs when $\phi_{l}^{11}$ is given by Eq. (5.1). Only if $\phi_{l}^{21}$ is given by

$$
\phi_{l}^{21}=\frac{\pi}{2}
$$

as well do they coincide.

At a scattering resonance in the partial wave $l_{r}$, using Eqs. (2.11) for the $a_{l}$ polarization, we have

$$
t_{l}^{1}=\frac{(n) \partial_{l}(y)}{\mathcal{N}_{l}(x)}
$$

and

$$
t_{l}^{2}=\frac{(1) \mathcal{N}_{l}(x)}{\partial_{l}(y)}
$$

and, using

$$
\begin{aligned}
& \partial_{l}(y) \approx \mathcal{N}_{l}^{\prime}(y), \\
& g_{l}^{\prime}(y) \approx-\mathcal{N}_{l}(y),
\end{aligned}
$$

since high in the edge region

$$
x \lesssim l<y,
$$

we also obtain

$$
t_{l}^{4} \approx \frac{\partial_{l}(y)}{(1) \mathcal{N}_{l}(x)}\left[(1)^{2} \partial_{l}(x) \mathcal{N}_{l}(x)+(n)^{2}{\gamma_{l}^{\prime}}_{l}(x) \mathcal{N}_{l}^{\prime}(x)\right]
$$

For the $b_{l}$ polarization, the expressions for $t_{l}^{1}, t_{l}^{2}$, and $t_{l}^{4}$ are identical except for the replacement $(1) \leftrightarrow(n)$. For partial waves satisfying relation (6.7), we have $\mathrm{e}^{31}$

$$
\begin{aligned}
& \partial_{l}(y) \sim \mathcal{O}(1), \\
& \partial_{l}(x) \approx \frac{1}{2}\left(\frac{2 \Delta}{x}\right)^{-1 / 4} \exp \left[-\frac{x}{3}\left(\frac{2 \Delta}{x}\right)^{3 / 2}\right],
\end{aligned}
$$




$$
\begin{aligned}
& \mathcal{N}_{l}(x) \approx-\left(\frac{2 \Delta}{x}\right)^{-1 / 4} \exp \left[\frac{x}{3}\left(\frac{2 \Delta}{x}\right)^{3 / 2}\right] \\
& \mathcal{J}_{l}^{\prime}(x) \approx\left(\frac{2 \Delta}{x}\right)^{1 / 2} \partial_{l}(x) \\
& \mathcal{N}_{l}^{\prime}(x) \approx-\left(\frac{2 \Delta}{x}\right)^{1 / 2} \mathcal{N}_{l}(x),
\end{aligned}
$$

where

$$
\Delta=l-x .
$$

Substituting these approximations into Eqs. (6.4) and (6.5) and relation (6.8) and into Eqs. (2.30) an (2.31) at the scattering-resonance condition of Eq. (6.1), we obtain

$$
\tan \phi_{l}^{11} \sim\left(\frac{2 \Delta}{x}\right)^{1 / 2} \exp \left[\frac{-4 x}{3}\left(\frac{2 \Delta}{x}\right)^{3 / 2}\right]
$$

and

$$
\tan \phi_{l}^{21} \sim \exp \left[\frac{2 x}{3}\left(\frac{2 \Delta}{x}\right)^{3 / 2}\right] .
$$

For scattering resonances in partial waves, where $\Delta \gg 1$ [e.g., for $x=7446.95$, corresponding to the droplet radius of Eqs. (4.19) and (4.20), $\Delta \gtrsim 20$ ] we obtain $\phi_{l}^{11} \approx 0$ and $\phi_{l}^{21} \approx \pi / 2$. Thus scattering resonances in the upper portion of the edge region are nearly internal-reflection resonances as well. They correspond to the resonant partial wave's reflecting internally time after time inside the droplet with a constructive interference path length that produces an integer cycle phase delay. These are exactly the conditions under which a plane wave incident upon a square barrier for the flatinterface problem has a transmission resonance. ${ }^{27}$

In the partial-wave sums for the scattering amplitudes of Eqs. (2.4) and (2.5), we can separate the contribution of a resonant partial wave $l_{r}$ from all the others in the sum, which we call the background. The phases of the partial-wave amplitudes $a_{l}$ and $b_{l}$ in the background sum are distributed uniformly, and thus $\left(a_{l}\right)_{\text {ave }} \approx\left(b_{l}\right)_{\text {ave }} \approx 1 / 2$. Since the normalized scattering efficiency,

$$
\epsilon=\frac{2}{x^{2}} \sum_{l=1}^{l_{\max }}(2 l+1)\left(\left|a_{l}\right|^{2}+\left|b_{l}\right|^{2}\right),
$$

is proportional to the square of the magnitude of the partialwave scattering amplitudes, $l_{r}$ contributes to the scattering efficiency independently of the phases of the rest of the partial waves in the background sum. The situation is different for the angular scattering, since the scattering functions are sums over $a_{l}$ and $b_{l}$ and the phases of all the partialwave scattering amplitudes superpose with one another in these sums. In particular, the phase of $l_{r}$ superposes with the phase of the background sum in producing the observed angular scattering. If the phase of the background sum and the phase of $l_{r}$ are equal, as is the case for forward scattering, where

$$
S_{1}=S_{2}=\frac{1}{2} \sum_{l=1}^{l_{\max }}(2 l+1)\left(a_{l}+b_{l}\right),
$$

the effect that the resonant partial wave has on the angular scattering is enhanced. If the phase of the background sum destructively interferes with that of $l_{r}$, then the effect that the resonant partial wave has on the angular scattering is diminished. As the observation angle is varied from 0 to 180 $\mathrm{deg}$, the effect that the scattering resonance has on the angular scattering is variable as well.

In the Fourier transform of the scattering functions of relations (3.9)-(3.11), the width of the observer's aperture function allows one to control the width of the sinc functions and thus to control the number of partial waves in the background sum with which the phase of $l_{r}$ coherently interferes. Thus the Fourier transform measures not only the strength of a scattering resonance but also the nature of its interference with neighboring partial waves. This proves to be useful in that, since $l_{r}$ can be made to interfere with only a limited range of $l$ as opposed to the entire range of $l$ as in Eqs. (2.4) and (2.5), one can produce cooperative effects between scattering resonances and localized regions of interesting phase variation in the background sum, such as geometrical rays and families of internal-reflection resonances. This phase cooperation is examined in Section 7.

\section{NON-DEBYE GLARE SPOTS AND THE COOPERATION OF PARTIAL WAVES}

For the parameters of Eqs. (4.19)-(4.21), the partial wave $l_{r}$ $=7470$ resonates for the $a_{l}$ polarization, and $l_{r}=7457$ resonates for the $b_{l}$ polarization. For unpolarized incident light [Eq. (3.12)] and for $\theta_{0}=90 \mathrm{deg}$, the magnitude squared of the Fourier transform of the Mie-scattering amplitude is shown in Fig. 3. In the calculation of $F_{i}$, the Mie-scattering amplitude was computed by using the algorithm of Ref. 13, and a 512-point grid was used in the numerical evaluation of the Fourier-transform integral. This grid spacing gave a $\mathrm{Ny}$ quist frequency for $F_{i}$ of $349.7 \mathrm{deg}^{-1}$. The structure of $F_{i}$ at the edge of the droplet was found not to change when the Nyquist frequency was doubled. The glare spot $G$ is the direct-reflection glare spot corresponding to the second term of relation (3.11). The glare spots $T, R_{a}^{(2)}$, and $R_{a}^{(4)}$ are the transmitted geometrical-ray glare spot and the two- and four-internal-reflection geometrical-ray glare spots corre-

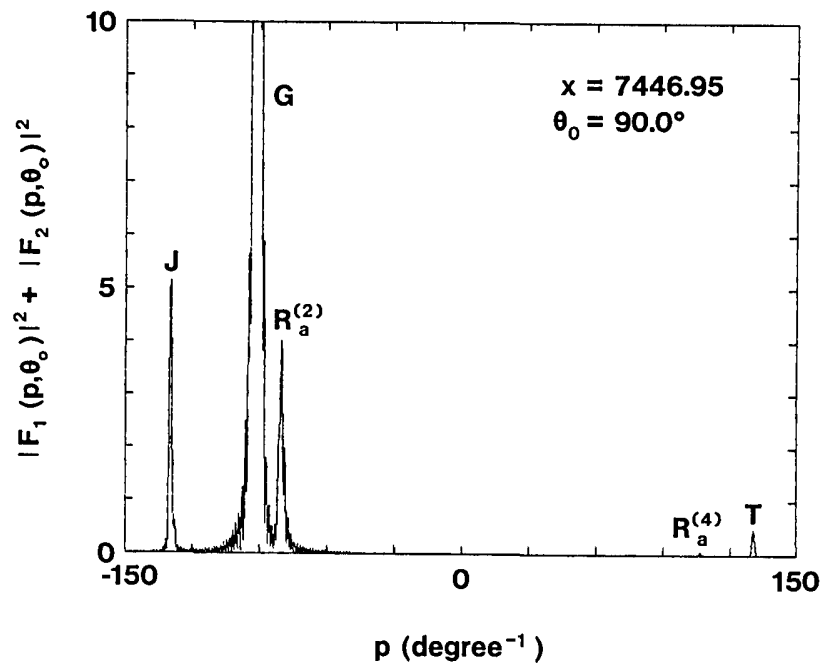

Fig. 3. 'T'he magnitude squared of the Fourier transform of the Mie electric field as a function of spatial frequency for $\theta_{0}=90 \mathrm{deg}$, partial waves 7457 and 7470 on resonance, and initially unpolarized light. 


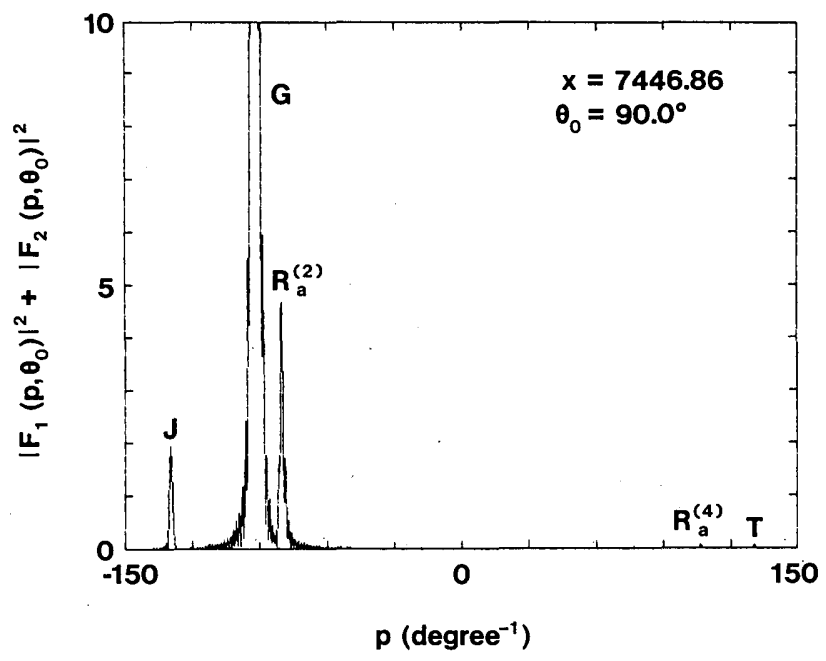

Fig. 4. Magnitude squared of the Fourier transform of the Mie electric field as a function of spatial frequency for $\theta_{0}=90 \mathrm{deg}$ partial waves 7457 and 7470 off resonance, and initially unpolarized light.

sponding to the $m=0,2,4$ portions of the third term of relation (3.11). The glare spot $J$ in Fig. 3 is the non-Debye glare spot. It occurs at the observation angle and spatial frequency of the 11th-order geometrical glare spot $R_{b}^{(11)}$, but its strength is extremely dependent on the size parameter, as may be seen in Fig. 4, for which $a=749.997 \mu \mathrm{m}$ and $x=$ 7446.86. For this size parameter, $l=7457$ and $l=7470$ are far off resonance.

It would thus seem that the glare spot $J$ is related both to scattering resonances and to the 11th-order rainbow. This is corroborated in Figs: 5 and 6, in which the area under the $J$ peak,

$$
A_{i}\left(\theta_{0}\right)=\int_{-x-\sigma}^{-x+\sigma} \mathrm{d} p\left|F_{i}\left(p, \theta_{0}\right)\right|^{2}
$$

with $\sigma=10.0 \mathrm{deg}^{-1}$, is plotted as a function of observation angle for the $S_{1}$ and $S_{2}$ polarizations, respectively. The $m=$ 11 Debye term contribution to $A_{i}$ was calculated from Eqs. (2.32) and (3.1) and is also shown in Figs. 5 and 6 . As is expected, the $m=11$ term peaks near $\theta_{\text {rainbow }}^{(11)}$, as described in Section 4, and the 11th-order rainbow is polarized strongly in the $S_{1}$ polarization. The 11 th-order rainbow provides a background upon which the effects of scattering resonances occur. This is shown in Fig. 7, in which $A_{2}\left(\theta_{0}=90 \mathrm{deg}\right)$ is plotted as a function of the size parameter $x$ for $7446.6 \leq x \leq$ 7447.3. The strong enhancements in $A_{2}$ are correlated well with the scattering resonances of $a_{l}$. The full set of scattering resonances for $7446 \leq l \leq 7530$ in this interval for the $a_{l}$ polarization is given in Table 1 .

The glare spot $J$ in Figs. 3 and 4 has a more complicated interpretation than do the other glare spots, $G, T, R_{a}^{(2)}$, and $R_{a}^{(4)}$, which are produced solely by geometrical rays. The observation angle $\theta_{0}$ and the spatial frequency $p$ of the glare spot $J$ may be explained in terms of the geometrical-ray glare spot $R_{b}^{(11)}$. However, the strength of the glare spot $R_{b}^{(11)}$ is size-parameter independent and is totally insufficient to explain the strong size-parameter dependence of $A_{i}$. The 11th-order rainbow provides only a background for the $J$ peak. Scattering resonances are responsible for the sizeparameter dependence of the strength of the $J$. They cause $A_{i}$ to grow to as much as $200 \%$ of the $R^{(11)}$ background in the $S_{1}$ polarization and to $285 \%$ of it in the $S_{2}$ polarization.

The large enhancements in the strength of the glare spot $J$ compared with the strength of the 11th-order rainbow are due to constructive interference between the resonant-partial-wave amplitude and, the 11th-order rainbow amplitude in relation (3.11). The portion of $F_{i}$ that is due to the $m$ order Debye term is

$$
\begin{aligned}
F_{i}^{(m)}\left(p, \theta_{0}\right)= & \frac{K}{2} \sum_{l=1}^{l_{\max }}\left[1-\left(r_{l}^{11}\right)^{2}\right]\left(r_{l}^{11}\right)^{m} \exp \left[i\left(2 \phi_{l}^{21}+m \phi_{l}^{11}-\pi\right)\right] \\
& \times\left\{\exp \left(i l \theta_{0}\right) \operatorname{sinc}\left[(p-l) \frac{\theta_{w}}{2}\right]\right. \\
& \left.+\exp \left(-i l \theta_{0}\right) \operatorname{sinc}\left[(p+l) \frac{\theta_{w}}{2}\right]\right\}
\end{aligned}
$$

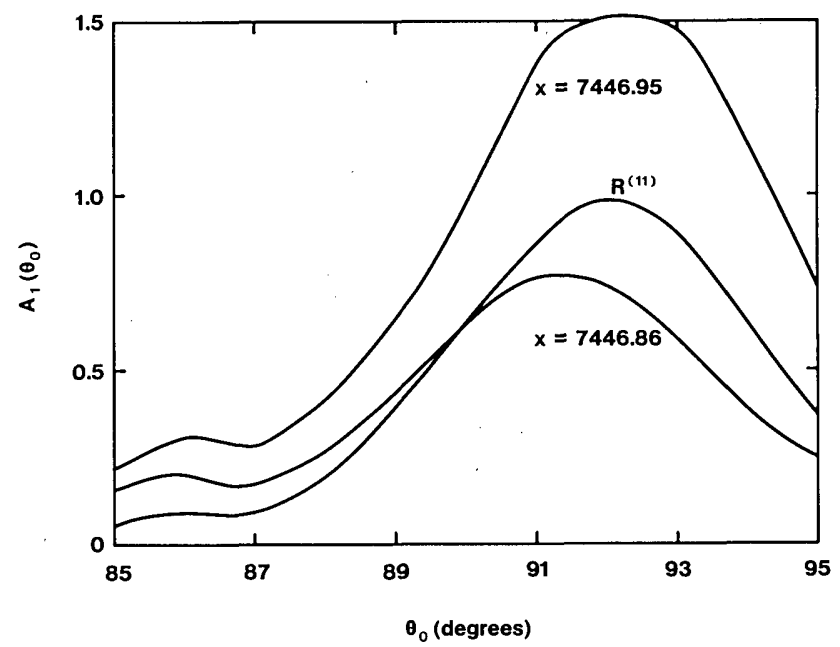

Fig. 5. Area under the non-Debye enhancement in the $S_{1}$ polarization as a function of observation angle. The contribution provided by the 11 th-order Debye term is denoted by $R^{(11)}$.

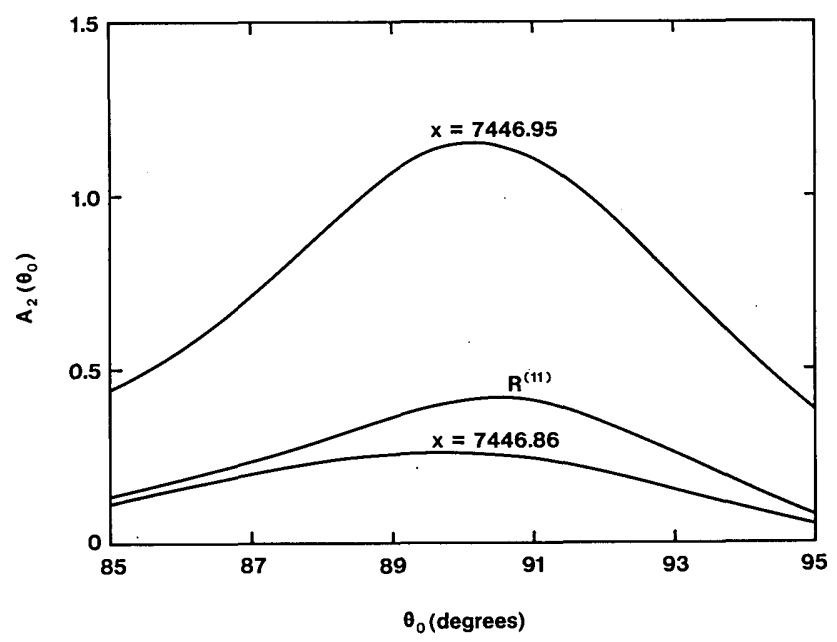

Fig. 6. Area under the non-Debye enhancement in the $S_{2}$ polarization as a function of observation angle. 


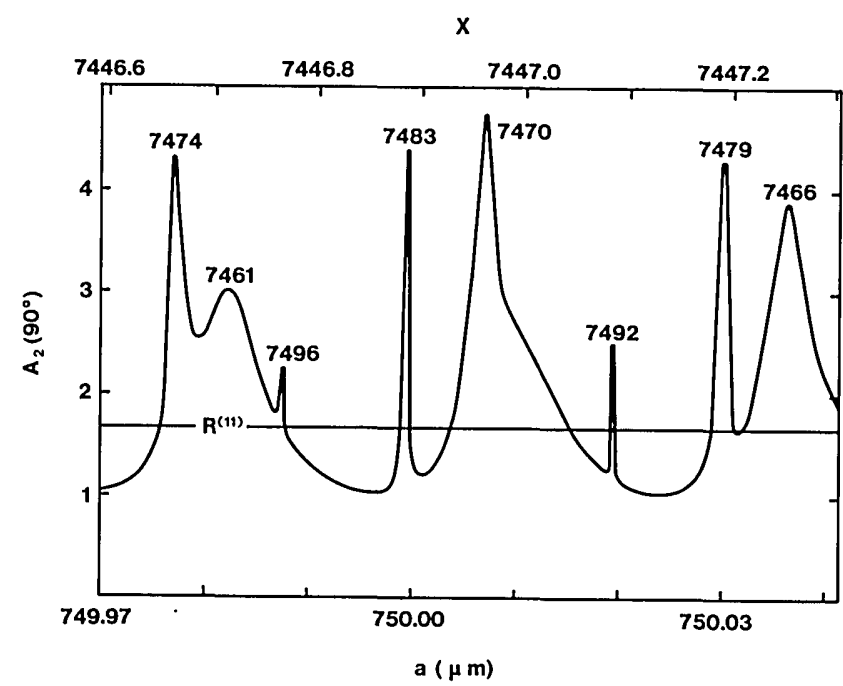

Fig. 7. Area under the non-Debye enhancement at $\theta_{0}=90 \mathrm{deg}$ as a function of droplet radius for the $S_{2}$ polarization. The contribution provided by the 11 th-order Debye term is denoted by $R^{(11)}$.

Table 1. Scattering Resonances for the $a_{l}$ Polarization $^{a}$

\begin{tabular}{ll}
\hline$l_{r}$ & $x$ \\
\hline 7448 & 7446.78 \\
7457 & 7447.00 \\
7461 & 7446.71 \\
7466 & 7447.24 \\
7470 & 7446.95 \\
7474 & 7446.66 \\
7479 & 7447.18 \\
7483 & 7446.88 \\
7492 & 7447.08 \\
7496 & 7446.76 \\
7501 & 7447.26 \\
7505 & 7446.93 \\
7509 & 7446.60 \\
7514 & 7447.09 \\
7518 & 7446.75 \\
7523 & 7447.23 \\
7527 & 7446.89 \\
\hline
\end{tabular}

${ }^{a}$ For $7446 \leq l \leq 7530$ and $7446.6 \leq x \leq 7447.3$.

and the portion of $F_{i}$ that is due to the resonant partial wave $l_{r}$ is

$$
\begin{aligned}
F_{i}^{\text {resonance }}\left(p, \theta_{0}\right)= & K\left\{\exp \left(i l_{r} \theta_{0}\right) \operatorname{sinc}\left[\left(p-l_{r}\right) \frac{\theta_{w}}{2}\right]\right. \\
& \left.+\exp \left(-i l_{r} \theta_{0}\right) \operatorname{sinc}\left[\left(p+l_{r}\right) \frac{\theta_{w}}{2}\right]\right\} .
\end{aligned}
$$

The phase of the contribution of the partial wave $l_{r}$ to the Fourier transform of the $m$-order Debye term is virtually the same as the phase of the $l_{r}$ scattering-resonance Fourier transform. This is because (i) a scattering resonance high in the edge region is also an internal-reflection resonance and (ii) for an internal-reflection resonance the phase of each term in the $m$ sum is the same as that of the entire $m$ sum itself. When the aperture function of the observer is of the order of a few degrees, it allows the $F_{i}^{\text {resonance }}$ amplitude to interfere coherently with the $F_{i}^{(m)}$ amplitude for a number of partial waves to either side of $l_{r}$. However, the upper portion of the edge region is not only the location of $l_{r}$. It is also the location of the orbiting-ray stationary-phase point $R_{c}^{(m)}$. Thus, for $\theta_{w}$, as in Eq. (3.12), since the phases of the $l_{\mathrm{r}}$ scattering resonance and the $R_{c}^{(11)}$ orbiting-ray stationary point are almost the same in the Fourier transform, the Fourier transform of the scattering-resonance amplitude constructively interferes with the Fourier transform of the 11-internal-reflection amplitude in the region of $R_{c}^{(11)}$. This constructive interference amplifies the effect that the scattering resonance has on the $J$ glare spot. It does this in the same way that the forward-scattering amplitude was amplified by the constructive interference between the scatteringresonance phase and the phase of the background sum. When the size parameter is such that $l_{r}$ is off resonance, its contribution to $F_{i}$ is much less, and its phase no longer constructively interferes with the $R_{c}^{(11)}$ stationary-phase region. This phenomenon is the cooperation of partial waves in Mie scattering.

An additional cooperation further enhances the contribution of scattering resonances to the non-Debye peak $J$ when $l_{r}$ is not too much larger than $x$. In this range, approximate internal-reflection resonances are produced in a number of lower partial waves when $x$ is in a reasonably large neighborhood about the value for which $l_{r}$ resonates. They are produced as follows. For $l \gtrsim x, \Delta \phi_{l}^{11}$ is nearly constant. If $z$ is given by

$$
l=l_{r}+z,
$$

and if a positive integer $I$ can be found so that

$$
2 \pi I=z \Delta \phi_{l}^{11},
$$

then the phase $\phi_{l}^{11}$ advances through exactly $I$ cycles for every additional $|z|$ partial waves below $l_{r}$. This condition generates internal-reflection resonances in the lower partial waves, since the resonant partial wave $l_{r}$ itself is an internalreflection resonance. For example, when $x \approx 7446.95$ and $n$ $=1.331, \Delta \phi_{l}^{11} \approx 82.74 \mathrm{deg}$ for the $a_{l}$ polarization, $\Delta \phi_{l}^{11} \approx 82.56$ deg for the $b_{l}$ polarization, and Eq. (7.5) is nearly satisfied for $|z|=13$ and $I=3$. Thus $\phi_{l}^{11}$ advances almost exactly 3 cycles every 13 partial waves. For size parameters in the neighborhood of the $x$ for which $l_{r}=7470$ resonates for the $a_{l}$ polarization, approximate internal-reflection resonances are produced in the partial waves $l=7405,7418,7431,7444,7457$. These are shown in Table 2. These partial waves possess approximate internal-reflection resonances for $7446.88 \leqslant x$ $\lesssim 744.7 .18$.

Although the $m$ sums for these lower $l$ partial-wave resonances have large magnitudes, as shown in Table 2, they merely act as part of the size-parameter-insensitive background and make no strong contribution to the Fourier transform of the scattering functions unless they constructively interfere with each other. This constructive interference occurs in the first sinc function in relation (3.11) for the observation angles

$$
\theta_{0}=\frac{(L-I) 2 \pi}{|z|}-2 \Delta \phi_{\mathrm{ave}}^{21}-\frac{\left(\gamma_{l_{r}}-\gamma_{l}\right)}{|z|}
$$

and it occurs in the second sinc function in relation (3.11) for 
Table 2. Magnitudes and Phases of Various Contributions to the Fourier Transform of the Scattering Amplitude ${ }^{a}$

\begin{tabular}{cccc}
\hline$l$ & Magnitude & Phase (deg) & $\phi_{l}^{11}(\mathrm{deg})$ \\
\hline $\begin{array}{c}\text { Scattering resonance } \\
7470\end{array}$ & 1.95 & & 0.20 \\
$\begin{array}{c}\text { Internal-reflection resonances } \\
7405\end{array}$ & 1.58 & 5.19 & -7.55 \\
7418 & 1.54 & 7.56 & -9.68 \\
7431 & 1.52 & 54.25 & -9.32 \\
7444 & 1.52 & 77.56 & -7.06 \\
7457 & 1.50 & 70.75 & -3.93 \\
7449 & & 27.13 & \\
7476 & 0.0512 & 26.06 & -5.69 \\
11 -internal-reflection stationary points & 0.0169 & & \\
\hline
\end{tabular}
${ }^{a}$ Data are shown for the conditions of $a_{l}$ polarization, $x=7446.95$, and $\theta_{0}=90.0$ deg. For the 11 -internal-reflection stationary points, the magnitude is that of
each partial wave at the stationary point.

$$
\theta_{0}=\frac{(L+I) 2 \pi}{|z|}+2 \Delta \phi_{\mathrm{ave}}^{21}+\frac{\left(\gamma_{l_{r}}-\gamma_{l}\right)}{|z|}
$$

where $L$ is an integer and where

$$
\Delta \phi_{\mathrm{ave}}^{21}=\frac{\phi_{l_{r}}^{21}-\phi_{l}^{21}}{|z|} .
$$

It can be shown that these observation angles are within a few degrees of the scattering angles of the large- $m$ critically refracted rays. Thus, in the angular intervals in which the $R_{b}^{(m)}$ and $R_{c}^{(m)}$ stationary-phase regions are in the process of separating, the members of the dominant family of internalreflection resonances constructively interfere with one another and with the $R^{(m)}$ phases. This additional constructive interference somewhat strengthens the $J$ peaks at these observation angles for all size parameters. At the particular size parameter for which the $l_{r}$ scattering resonance occurs, its large-magnitude contribution to the Fourier transform of the scattering functions not only constructively interferes with the $R_{c}^{(m)}$ stationary-phase region but also generally constructively interferes with the family of internal-reflection resonances that is produced by means of Eq. (7.5). For the example of $l_{r}=7470$ for the $a_{l}$ polarization, this constructive interference is apparent in the phases given in Table 2. This additional constructive interference enhances further the contribution of the scattering resonance to the $J$ peak.

Finally, in this paper I have concentrated on one polarization of the incident light, on scattering resonances in one narrow range of size parameters, and on one narrow range of observation angles about the 11th-order rainbow. The mechanism discussed here has a more general validity. Scattering resonances occur over the entire range of droplet sizes. Thus, as an individual droplet grows or evaporates, the $J$ peaks in $\left|F_{i}\right|^{2}$ persist because different resonances cooperate one after the other with the $R_{c}^{(m)}$ stationary-phase points. There are $J$ peaks at observation angles near all the large- $m$ critical refraction scattering angles $\theta_{c}^{(m)}$. The $J$ enhancement occurs for both the $S_{1}$ and the $S_{2}$ polarizations. But since rainbows are polarized strongly in the $S_{1}$ polarization, the enhancement-to-background ratio is larger in the $S_{2}$ polarization. Experiments designed to observe the $J$ non-Debye enhancements for the $S_{2}$ polarization are now in progress. ${ }^{32}$

\section{REFERENCES}

1. H. M. Nussenzveig, "The theory of the rainbow," Sci. Am. 236, 116-127 (1977).

2. H. C. van de Hulst, Light Scattering by Small Particles (Dover, New York, 1957), Sec. 13.23.

3. C. W. Querfeld, "Mie atmospheric optics," J. Opt. Soc. Am. 55, 105-106 (1965).

4. C. F. Bohren and D. R. Huffman, Absorption and Scattering of Light by Small Particles (Wiley, New York, 1983), Sec. 4.3.3.

5. H. M. Nussenzveig, "High frequency scattering by a transparent sphere. II. Theory of the rainbow and the glory," J. Math. Phys. 10, 125-176 (1969).

6. J. Walker, "Multiple rainbows from single drops of water and other liquids," Am. J. Phys. 44, 421-433 (1976).

7. J. Lock, "The theory of the observations made of high order rainbows from a single water droplet," Appl. Opt. 26, 5291-5298 (1987).

8. H. M. Nussenzveig, "High frequency scattering by a transparent sphere. I. Direct reflection and transmission," J. Math. Phys. 10, 82-124 (1969).

9. Ref. 2 , Sec. 12.35 .

10. Ref. 2, Sec. 12.32 .

11. E. Hecht, Optics, 2nd ed. (Addison-Wesley, Reading, Mass., 1987), Sec. 9.6.

12. J. R. Reitz, F. J. Milford, and R. W. Christie, Foundations of Electromagnetic Theory, 3rd ed. (Addison-Wesley, Reading, Mass., 1979), Sec. 18.5.

13. W. J. Wiscombe, "Improved Mie scattering algorithms," Appl. Opt. 19, 1505-1509 (1980).

14. Ref. 2, Sec. 12.31.

15. A. Messiah, Quantum Mechanics (Wiley, New York, 1968), Sec. 10.9 .

16. Ref. 2, Sec. 12.33 .

17. J. R. Probert-Jones, "Surface waves in backscattering and the localization principle," J. Opt. Soc. Am. 73, 503 (1983).

18. M. Minnaert, The Nature of Light and Color in the Open Air (Dover, New York, 1954) Sec. 125.

19. K. W. Ford and J. A. Wheeler, "Semiclassical description of scattering," Ann. Phys. (NY) 7, 259-286 (1959).

20. V. Khare and H. M. Nussenzveig, "The theory of the glory," in Statistical Mechanics and Statistical Methods in Theory and Application, U. Landman, ed. (Plenum, New York, 1977), pp. 723-764.

21. P. R. Conwell, P. W. Barber, and C. K. Rushforth, "Resonant spectra of dielectric spheres," J. Opt. Soc. Am. A 1, 62-67 (1984).

22. P. Chýlek, J. D. Pendleton, and R. G. Pinnick, "Internal and near-surface scattered fields of a spherical particle at resonant conditions," Appl. Opt. 24, 3940-3942 (1985).

23. P. Chýlek, "Partial wave resonances and the ripple structure in the Mie normalized extinction cross section," J. Opt. Soc. Am. 66, 285-287 (1976).

24. P. Chýlek, J. T. Kiehl, and M. K. W. Ko, "Narrow resonance 
structure in the Mie scattering characteristics," Appl. Opt. 17, 3019-3021 (1978).

25. P. Chýlek, J. T. Kiehl, and M. K. W. Ko, "Optical levitation and partial wave resonances," Phys. Rev. A 18, 2229-2233 (1978).

26. J. R. Probert-Jones, "Resonance component of backscattering by large dielectric spheres," J. Opt. Soc. Am. A. 1, 822-830 (1984).

27. E. Merzbacher, Quantum Mechanics, 2nd ed. (Wiley, New York, 1970), Sec. 6.8.

28. J. A. Lock, "The temporary capture of light by a dielectric film," Am. J. Phys. 53, 968-971 (1985).
29. V. Khare and H. M. Nussenzveig, "Theory of the glory," Phys. Rev. Lett. 38, 1279-1282 (1977).

30. H. M. Nussenzveig, "Complex angular momentum theory of the rainbow and the glory," J. Opt. Soc. Am. 69, 1068-1079, 11931194 (1979).

31. M. Kerker, The Scattering of Light and Other Electromagnetic Radiation (Academic, New York, 1969), Sec. 3.6.1.

32. J. A. Lock and J. R. Woodruff, "Non-Debye enhancements in the Mie scattering of light from a single water droplet," submitted to Appl. Opt. 